LA W RENCE LIVERMORE NATIONAL LABORATORY
Gamma ray imaging of the imploding shell using NIS hardware

N. Izumi, R. Tommasini

June 14, 2013 
This document was prepared as an account of work sponsored by an agency of the United States government. Neither the United States government nor Lawrence Livermore National Security, LLC, nor any of their employees makes any warranty, expressed or implied, or assumes any legal liability or responsibility for the accuracy, completeness, or usefulness of any information, apparatus, product, or process disclosed, or represents that its use would not infringe privately owned rights. Reference herein to any specific commercial product, process, or service by trade name, trademark, manufacturer, or otherwise does not necessarily constitute or imply its endorsement, recommendation, or favoring by the United States government or Lawrence Livermore National Security, LLC. The views and opinions of authors expressed herein do not necessarily state or reflect those of the United States government or Lawrence Livermore National Security, LLC, and shall not be used for advertising or product endorsement purposes.

This work performed under the auspices of the U.S. Department of Energy by Lawrence Livermore National Laboratory under Contract DE-AC52-07NA27344. 
Date: June 4, 2013

Written by: Nobuhiko IZUMI (izumi2@IInl.gov), Riccardo Tommasini (tommasini2@IInl.gov)

Prepared by LLNL under Contract DE-AC52-07NA27344.

Memo

\section{Gamma ray imaging of the imploding shell using NIS hardware}

\section{(1) Background}

Imaging 4.433 MeV gamma ray from $12 \mathrm{C}\left(n, n^{\prime} \gamma\right)$ reactions would make it possible to measure shape of the imploded shell at maximum neutron emission time. This document studies the feasibility of imaging $4.433 \mathrm{MeV}$ gamma rays with using existing NIS by setting the gate time on the arrival timing of the gamma rays.

\section{(2) Benefits of gamma-ray imaging}

1. No need for diagnostic holes on the hohlraum

2. No contamination due to self-emission from the hot core

3. No need to sacrifice drive beams for backlighting

4. Interpretation of the image is straight forward compare to the scattered neutron images because of isotropic emission of $12 C\left(n, n^{\prime} g\right)$

5. Can still live with existing primary or downshifted neutron imager

\section{(3) Summary}

Imaging of the $4.433 \mathrm{MeV}$ gamma ray from $12 \mathrm{C}\left(\mathrm{n}, \mathrm{n}^{\prime} \gamma\right)$ is doable with using existing NIS when $\mathrm{Yn}$ $>1 \mathrm{E} 15$ and rhoR_CH $>300 \mathrm{mg} / \mathrm{cm} 2$ with the detection statistics of $~ 84$ events/resolution element. The image quality can be significantly improved (to 285 count/resolution element) by replacing existing plastic scintillator array (thickness $5 \mathrm{~cm}$ ) with new Lu2SiO5 (LSO) scintillator array (thickness $2.5 \mathrm{~cm}$ ).

Table 1. Quality of $4.433 \mathrm{MeV}$ gamma ray image obtainable with existing NIS

\begin{tabular}{|ccc|}
\hline Scintillator array & Plastic & LSO \\
\hline Spatial resolution & 30um at object & 30um at object \\
\hline Pinhole diameter & $20 \mathrm{um}$ & $20 \mathrm{um}$ \\
\hline Detector resolution FWHM & $2 \mathrm{~mm}$ & $2 \mathrm{~mm}$ \\
\hline Detection efficiency & $\sim 14 \%$ & $\sim 50 \%$ \\
\hline Magnification & 92.4 & 92.4 \\
\hline Scintillator Thickness & $5 \mathrm{~cm}$ & $2.5 \mathrm{~cm}$ \\
\hline $\begin{array}{c}\text { Detected } \gamma / \text { res_elem } \\
\text { Yn(DT) }=1 \mathrm{E} 5, \rho \Delta R=300 \\
\mathrm{mg} / \mathrm{cm}^{2}\end{array}$ & 84 & 285 \\
\hline Scintillation efficiency & & \\
\hline
\end{tabular}




\section{(4) Production of gamma-ray in the imploded shell}

Fig. 1 shows cross section of the $12 C\left(n, n^{\prime} \gamma\right)$ reactions as a function of neutron energy. The production of $12 C\left(n, n^{\prime} \gamma\right)$ is calculated by the equation:

$$
\begin{aligned}
& \mathrm{Y}_{\gamma}:=\left(1-\exp \left(-\frac{\sigma_{\mathrm{C}}}{\mathrm{m}_{\mathrm{C}}+\mathrm{m}_{\mathrm{H}}} \cdot \rho \Delta \mathrm{R}\right)\right) \cdot \mathrm{Yn}=2.91 \times 10^{12} \text { photons } \\
& \rho \Delta \mathrm{R}=300 \frac{\mathrm{mg}}{\mathrm{cm}^{2}} \\
& \sigma_{\mathrm{C}}=0.21 \mathrm{barn} \\
& \mathrm{Yn}=1 \times 10^{15} \text { neutrons }
\end{aligned}
$$

The branching ratio of $t(d, \gamma)$ reaction is about $5 \mathrm{E}-5(\mathrm{E} \gamma: 16.7 \mathrm{MeV})$. The expected number for the $12 \mathrm{C}\left(\mathrm{n}, \mathrm{n}^{\prime} \gamma\right)$ yield is $\sim 60$ times more than that of self-emission gamma-ray from $t(d, \gamma)$ reactions.

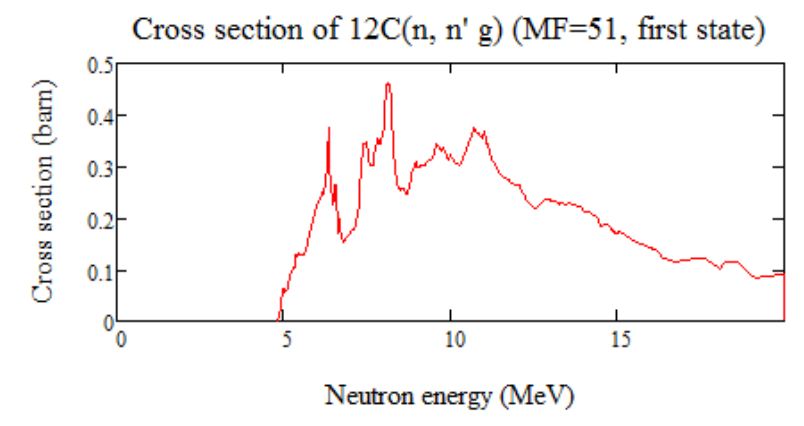

Fig. 1. ENDF/B-VI MOD 3 Evaluation, June 1996, M.B. Chadwick and P.G. Young (LANL) 


\section{(5) Photometrics (plastic scintillator)}

In what follows we approximate the imaging pinhole with a 20um diameter circular holes located $30 \mathrm{~cm}$ from the TCC.

Because the solid angle subtended by each pinhole is $\sim 3.5$ nano-steradian, the expected number of photons going through the pinhole is,

$$
\begin{aligned}
& N_{\text {inc }}:= \mathrm{Y}_{\gamma} \cdot \frac{\Omega_{\mathrm{PH}}}{4 \pi \mathrm{sr}}=808.334 \\
& \Omega_{\mathrm{PH}}=3.491 \times 10^{-9}
\end{aligned}
$$

The quantum efficiency of the scintillator array can be estimated by cross section ofthe Compton scattering and thickness of the scintillator (plastic, $5 \mathrm{~cm}$ ).

QE_scinti $:=1-\exp \left(-\Sigma_{-}\right.$scinti.t_scinti $)=13.587 \%$

Therefore the expected number of detected photon per pinhole is,

$$
\mathrm{N}_{\text {det }}:=\mathrm{QE} \_ \text {scinti }-\mathrm{N}_{\text {inc }}=110
$$

By adding up the images from 20 pinholes, we can increase the number of detected photons to 2200 events/image.

The size of the gamma ray image strongly depends on how the stagnation goes on the last 100ps to the neutron bang. Here we assume shell material exist on $\mathrm{R}=50 \sim 100 \mathrm{um}$ region. Then expected detected photons per resolution element is,

$$
\frac{\mathrm{Y}_{\gamma}}{4 \pi} \cdot \Omega_{\mathrm{PH}} \cdot 20 \cdot \mathrm{QE} \_ \text {scinti } \cdot \frac{(30 \mu \mathrm{m})^{2}}{\pi \cdot\left[(100 \mu \mathrm{m})^{2}-(50 \mu \mathrm{m})^{2}\right]}=84 \frac{\text { events }}{\text { res_elem }}
$$

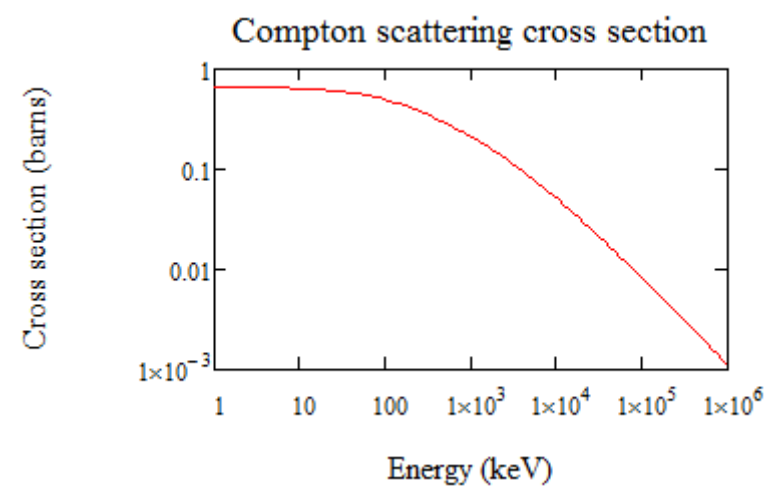

Fig. 2. Cross section of Compton scattering 
Simulated images with 2200 detected photons are shown below.

The extent of the images is 200um $\times 200 u m$. In this case the photon statistics is not rich but enough to extract low mode perturbation (P0 and P2) of the shell ( 84 events/res_elem).

Object

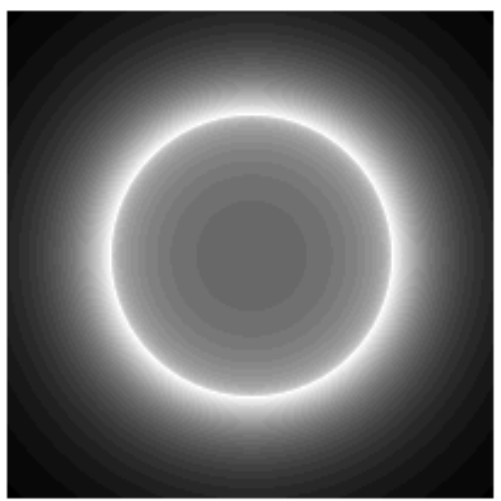

w/ poission noise

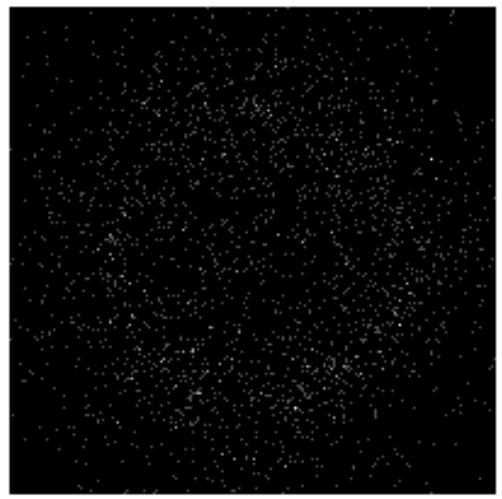

Smoothed by PSF

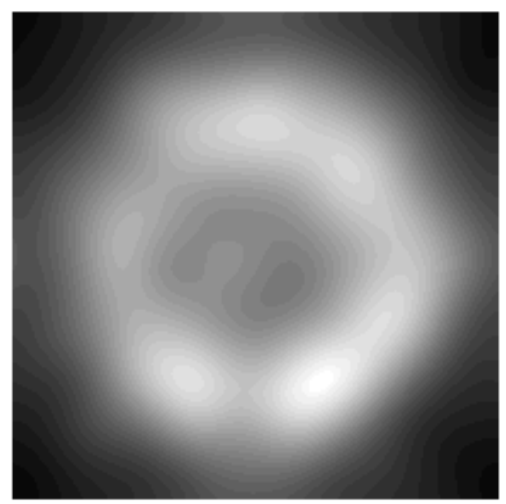

Fig. 3. Quality of gamma ray image expected for $Y n=1 E 15, \rho \Delta R=300 \mathrm{mg} / \mathrm{cm}^{2}$

With neutron yields increased by $4 x,(Y n \sim 4 E 15)$, or with ablator $\rho \Delta R$ increased by $4 X,(\rho \Delta R \sim$ $\left.1.2 \mathrm{~g} / \mathrm{cm}^{2}\right)$, the quality of the recorded image is improved to making it possible to extract intermediate modes (up to mode 6) of the shell (336 events/res_elem).

Object

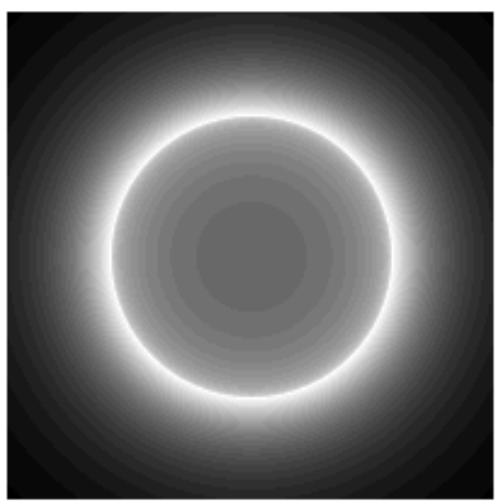

w/ poission noise

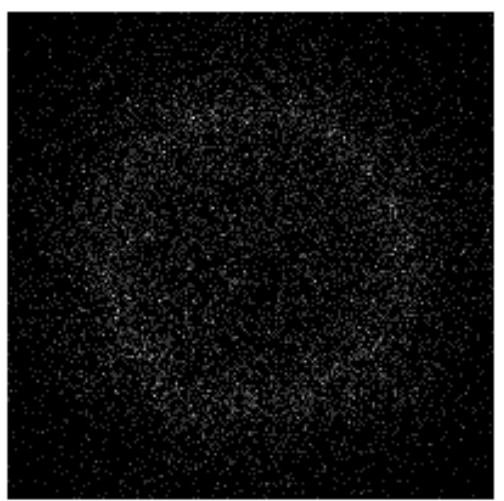

Smoothed by PSF

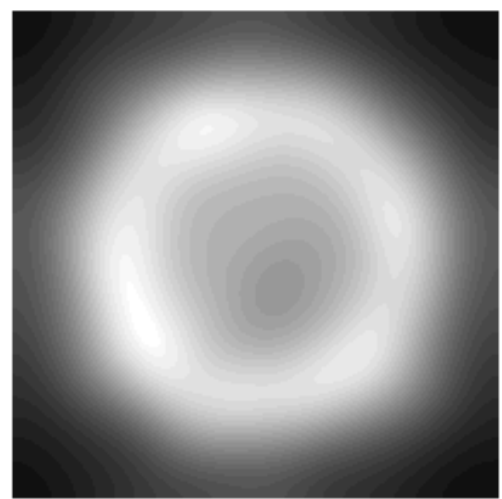

Fig. 4. Quality of gamma ray image expected for $Y n=4 E 15, \rho \Delta R=300 \mathrm{mg} / \mathrm{cm}^{2}$ 


\section{(6) Photometrics (LSO scintillator)}

One obvious way to improve the obtainable image quality is use of scintillator consists of high Z materials. Fig. 5 is the comparison of the quantum efficiencies for the plastic and the LSO scintillator. By replacing existing plastic scintillator array $(5 \mathrm{~cm}$ thick) with new LSO array $(2.5 \mathrm{~cm})$, it is possible to improve the quantum efficiency by factor 3.4. LSO is one of the most efficient inorganic scintillators. Typical scintillation efficiency of the LSO (3E4 photons/MeV) is factor 3 higher than typical plastic scintillators (1E4 photons/MeV). Fig. 6 . is showing the quality of the expected gamma ray image with the LSO scintillator array. It is possible to measure asymmetry of the imploded shell even on a cryo-layered shot with $\mathrm{Yn}=1 \mathrm{E} 15, \rho \Delta \mathrm{R}=300 \mathrm{mg} / \mathrm{cm}^{2}$ (image quality 285 events/res_elem). This technique is also applicable to DT filled SymCap shots.

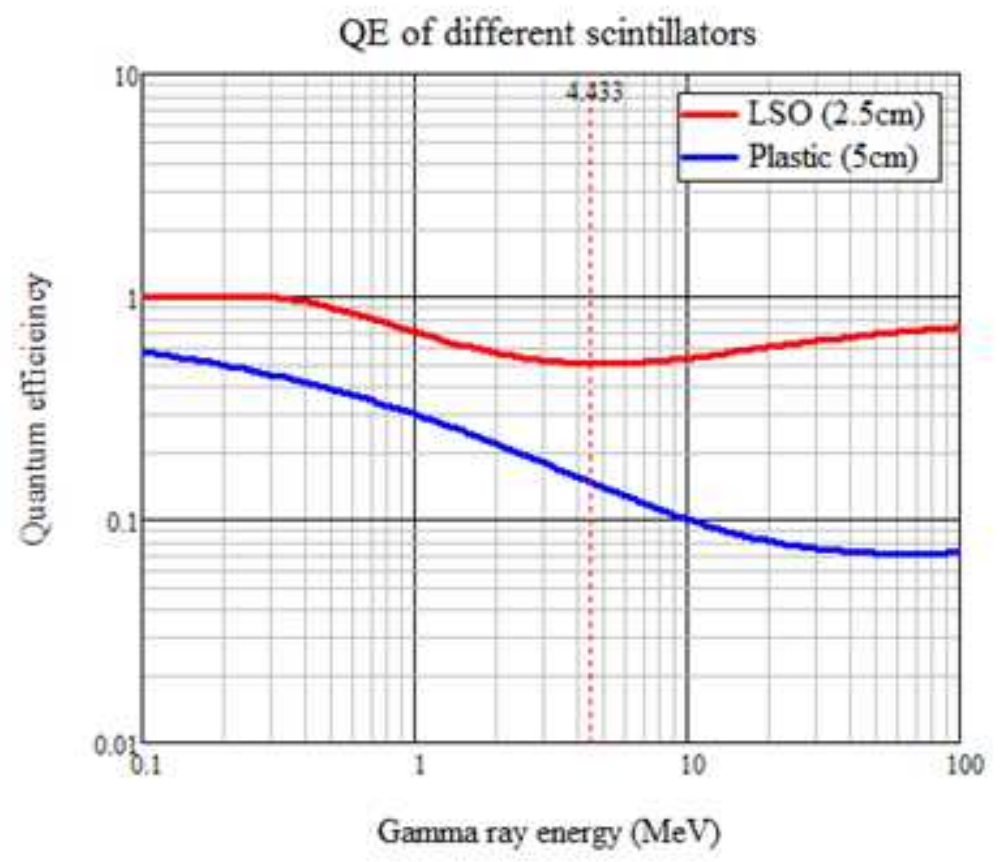

Fig. 5. Comparison of detection efficiency for LSO and plastic scintillator

Object

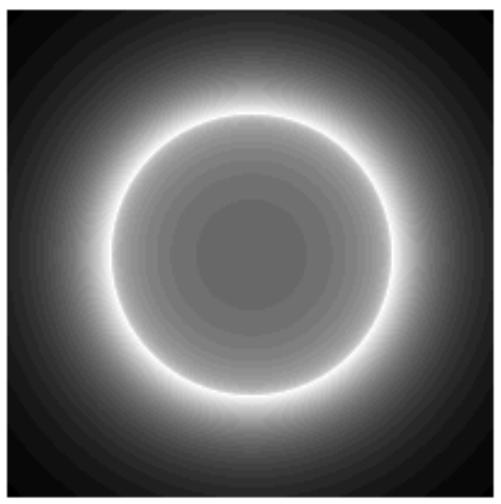

w/ poission noise

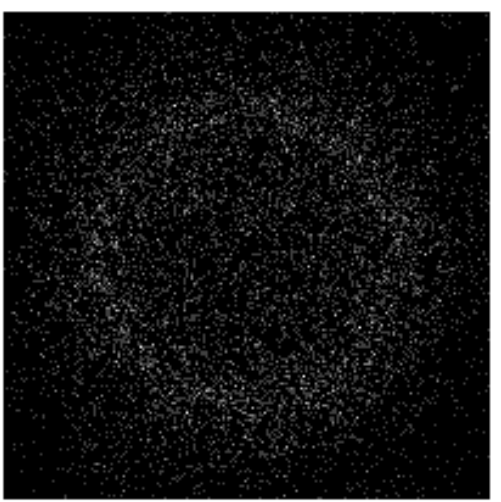

Smoothed by PSF

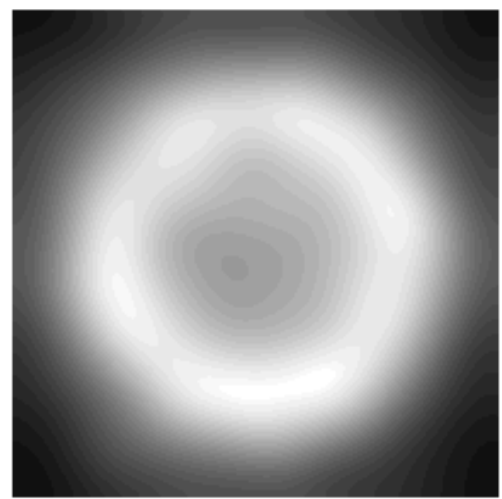

Fig. 6. Expected gamma ray image with LSO scintillator array $\left(\mathrm{Yn}=1 \mathrm{E} 15, \rho \Delta \mathrm{R}=300 \mathrm{mg} / \mathrm{cm}^{2}\right)$ 


\section{(7) intrinsic spatial resolution limit of the scintillator array (Plastic scintillator array)}

The existing plastic scintillator array of the NIS was designed for imaging of $14 \mathrm{MeV}$ neutrons. The expected spatial resolution of the image is affected by the PSF of the aperture and by the lateral range of the recoiled protons (or deuterons) in the scintillator array. If we use the same plastic scintillator array for $4.433 \mathrm{MeV}$ gammarays, the detector resolution is determined by the lateral range of the Compton electrons. In order to estimate the achievable spatial resolution of the existing plastic scintillator array, a Monte Carlo simulation was performed.

In this simulation, a pencil beam of $4.433 \mathrm{MeV}$ gammarays is hitting the plastic scintillator array. The gamma-rays are causing Compton scattering and generating high energy primary electrons. The trajectories of the energetic electrons are calculated using the stopping power for the electrons in the scintillator. The differential cross-section of the Compton scattering was used to determine the direction of the Compton electrons.

Fig. 7 is the angular distribution of the electrons. The angular distribution of the Compton electrons has a strong peak in the forward direction. The distribution has a zero at theta $=0$ simply because the cross section $(d \sigma / d \Omega)$ was weighted by $2 \pi \sin (\theta) d(\theta)$.

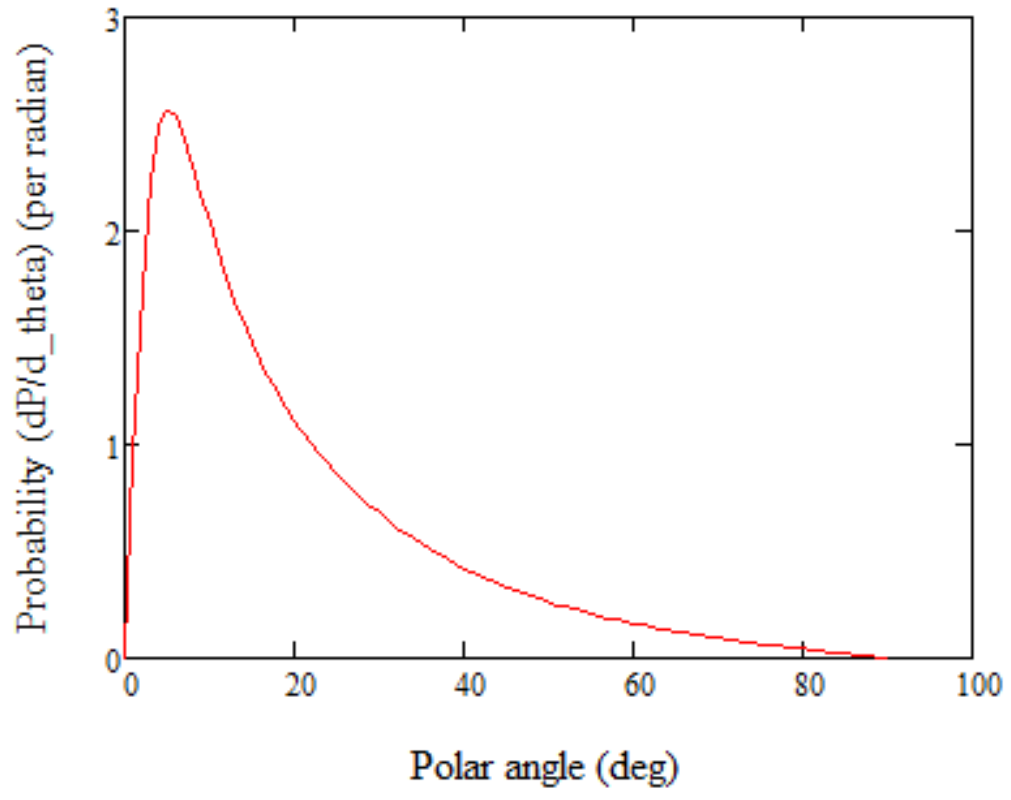

Fig. 7. Angular distribution of electrons produced by Compton scattering in the scintillators 
Fig. 8 shows the ray-trace of the knock-on electrons in the plastic scintillator. Units are $\mathrm{mm}$. As expected, the trajectories of the electrons are strongly peaked on the forward direction (Moliere scattering of the electrons was not included in this calculation).

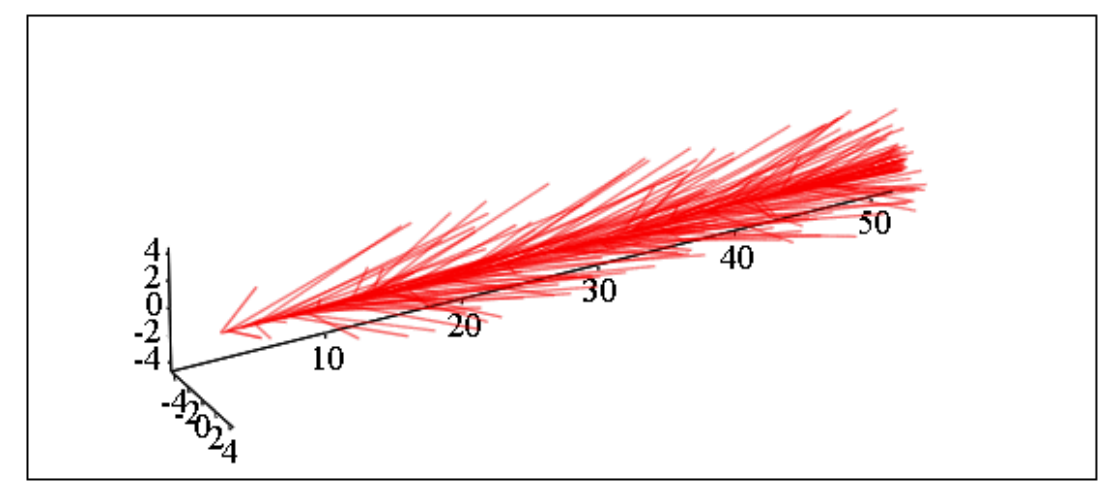

Fig. 8. Trajectories of electrons produced in the plastic scintillator array

Fig. 9 shows calculated point spread function of the plastic scintillator array. The slowdown of each electron was calculated using the total stopping power. The ionization of the scintillator was calculated using the collisional stopping power. Most of the electron energy is deposited within $1 \mathrm{~mm}$ from the gamma-ray path. Therefore even for Compton electrons having long stopping range is possible to obtain $\sim 2 \mathrm{~mm}$ spatial resolution on the detector. Because the expected magnification is $\sim 92 \mathrm{x}$, this $2 \mathrm{~mm}$ resolution on detector correspond to only $22 \mathrm{um}$ on the object. Accounting spatial resolution given by pinhole size (20um), the expected spatial resolution of the system is about 30 um.

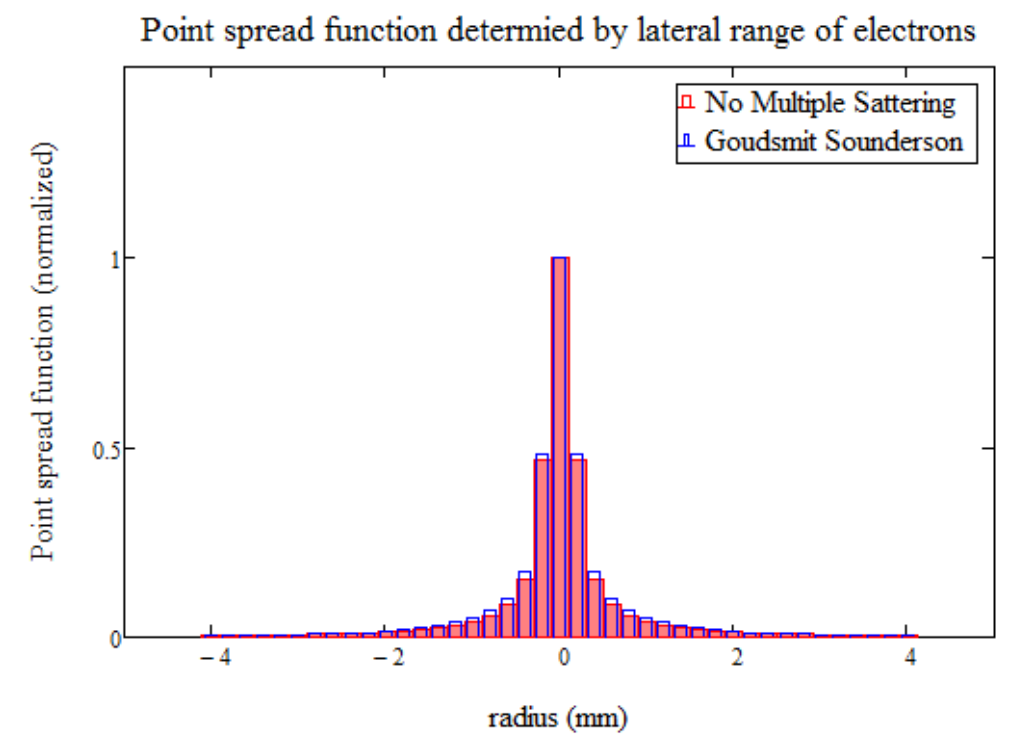

Fig. 9. Calculated spatial resolution of plastic scintillator array excited by $4.433 \mathrm{MeV}$ gamma ray 


\section{(8) Intrinsic spatial resolution limit of the scintillator array (LSO scintillator)}

Estimation of detector spatial resolution of the LSO scintillator is not trivial. Increased electron stopping power in the scintillator helps to reduce the range of the primary electrons. Howeverthe Moliere scattering (multiple scattering of electrons), x-ray fluorescence, and pair production/annihilation in the scintillator may affect the extent of energy deposition around the gammaray path. We performed a Monte Carlo simulation to assess spatial resolution of the LSO. The Goudsmit Saunderson model is used for the multiple scattering of the electrons. As shown in Fig. 10, the LSO scintillator showed spatial resolution similar to that of the plastic scintillator array. Most of gammaray energy is deposited less than $1 \mathrm{~mm}$ radius. Therefore it is possible to obtain $\sim 2 \mathrm{~mm}$ resolution on the LSO scintillator array.

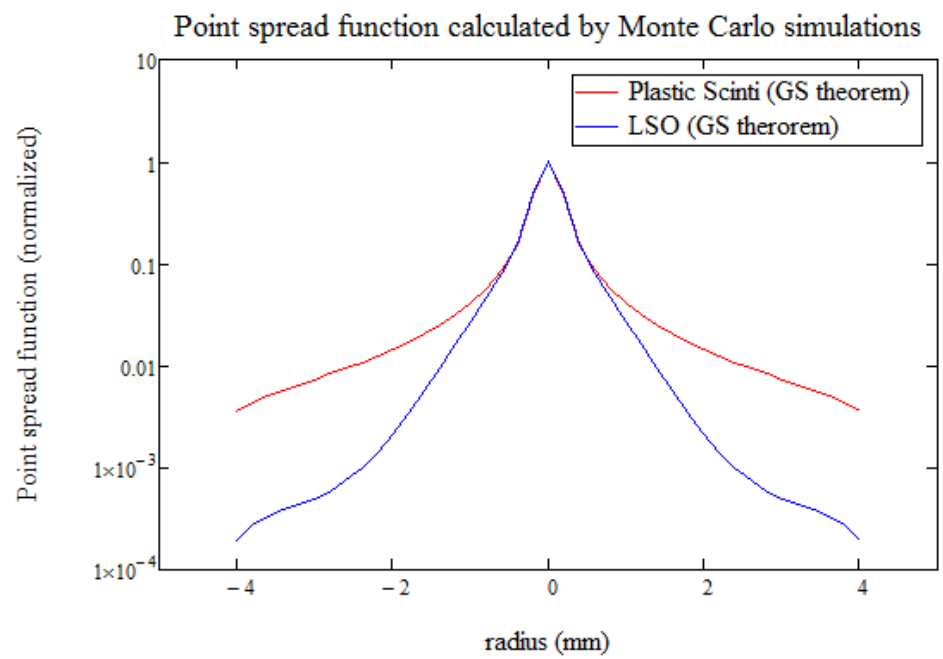

Fig. 10. Calculated point spread function for the plastic scintillator $(t \sim 5 \mathrm{~cm})$ and the $L S O(t \sim 2.5 \mathrm{~cm})$ 


\section{(9) Expected performance of the pinhole array designed for 4.433 MeV gamma-rays.}

Typical mean free path of $14 \mathrm{MeV}$ neutrons in a dense material like tungsten is $\sim 3 \mathrm{~cm}$. The mean free path of $4.433 \mathrm{MeV}$ gamma-rays in tungsten is $1.3 \mathrm{~cm}$. Therefore the pinhole array originally designed for neutron imaging will work for imaging of $4.433 \mathrm{MeV}$ gamma rays.

(10)

Flight time for the gamma-rays and neutrons

In order to obtain a gamma ray image, we need to gate-off the detector before the arrival of the primary neutrons. The respective time-of-flights are:

Neutrons: $2802.5 \mathrm{~cm} / 5.13(\mathrm{~cm} / \mathrm{ns})=546.6 \mathrm{~ns}$

Gamma: $2802.5 \mathrm{~cm} / \mathrm{c}=93.5 \mathrm{~ns}$

I.e., We have $\sim 450$ ns separation from the gamma ray arrival to the neutron arrivalat the detector. This separation is long compare to decay time of the LSO scintillator (50ns).

(11) Expected scintillation efficiency (Plastic scintillator)

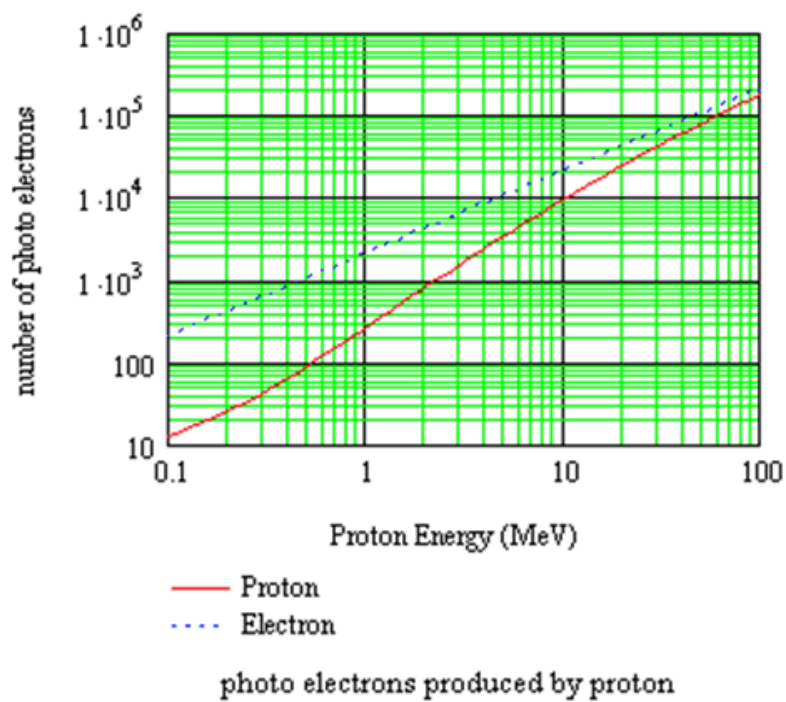

$$
\begin{aligned}
& \Delta \mathrm{L}(\mathrm{SP}):=\mathrm{S} \cdot\left(1+\mathrm{kB} 2 \mathrm{SP}+\mathrm{C} 2 \cdot \mathrm{SP}^{2}\right)^{-1} \\
& \frac{\Delta N}{\Delta E}=\frac{S}{1+k B \cdot\left(\frac{d E}{d X}\right)+C_{2} \cdot\left(\frac{d E}{d X}\right)^{2}} \\
& S=2113(\text { electrons } / \mathrm{MeV}) \\
& k B=1.29 \times 10^{-2} \frac{\mathrm{g} / \mathrm{cm}^{2}}{\mathrm{MeV}} \\
& C_{2}=9.59 \times 10^{-6}\left(\frac{\mathrm{g} / \mathrm{cm}^{2}}{\mathrm{MeV}}\right)^{2}
\end{aligned}
$$

The expected scintillation efficiency of a plastic scintillator is determined by the stopping range of the charged particles in the scintillator material. For the case of neutron detection, the scintillation efficiency is determined by the stopping power of the recoiled protons. For gamma ray imaging, the response is determined by the stopping power of the Compton electrons. According to the established model by R. L. Craun and D. L. Smith, the expected scintillation efficiency (per unit of absorbed energy) for gamma ray excitation is a factor 2 higher compared to the one for neutron excitation ( $E n=14 \mathrm{MeV}$, nominal recoiled proton energy $\sim 7 \mathrm{MeV}$ ). The reported scintillation efficiency of the LSO is even another factor 3 higher than the plastic scintillators. 